\title{
On the origin of brittle fracture of entangled polymer solutions and melts
}

\author{
Wagner, Manfred H.; Narimissa, Esmaeil; Huang, Qian
}

Published in:

Journal of Rheology

Link to article, DOI:

$10.1122 / 1.4995497$

Publication date:

2018

Document Version

Publisher's PDF, also known as Version of record

Link back to DTU Orbit

Citation (APA):

Wagner, M. H., Narimissa, E., \& Huang, Q. (2018). On the origin of brittle fracture of entangled polymer solutions and melts. Journal of Rheology, 62(1), 221-233. https://doi.org/10.1122/1.4995497

\section{General rights}

Copyright and moral rights for the publications made accessible in the public portal are retained by the authors and/or other copyright owners and it is a condition of accessing publications that users recognise and abide by the legal requirements associated with these rights.

- Users may download and print one copy of any publication from the public portal for the purpose of private study or research.

- You may not further distribute the material or use it for any profit-making activity or commercial gain

- You may freely distribute the URL identifying the publication in the public portal

If you believe that this document breaches copyright please contact us providing details, and we will remove access to the work immediately and investigate your claim. 


\title{
On the origin of brittle fracture of entangled polymer solutions and melts
}

\author{
Manfred H. Wagner ${ }^{a)}$ and Esmaeil Narimissa \\ Polymer Engineering / Polymer Physics, Berlin Institute of Technology (TU Berlin), Fasanenstrasse 90, \\ 10623 Berlin, Germany \\ Qian Huang \\ Department of Chemical and Biochemical Engineering, Technical University of Denmark, \\ 2800 Kongens Lyngby, Denmark
}

(Received 12 July 2017; final revision received 1 November 2017; published 28 November 2017)

\begin{abstract}
A novel criterion for brittle fracture of entangled polymer liquids is presented: Crack initiation follows from rupture of primary $\mathrm{C}-\mathrm{C}$ bonds, when the strain energy of an entanglement segment reaches the energy of the covalent bond. Thermal fluctuations lead to a short-time concentration of the strain energy on one $\mathrm{C}-\mathrm{C}$ bond of the entanglement segment, and the chain ruptures. This limits the maximum achievable stretch of entanglement segments to a critical stretch of $f_{c} \leq 6$. Recent experimental data of Huang et al. [Phys. Rev. Lett. 117, 087801 (2016)] ] and Huang and Hassager [Soft Matter 13, 3470-3474 (2017)] on fracture of solutions of nearly monodisperse polystyrenes dissolved in oligomeric styrene and of a well characterized polydisperse polystyrene melt, are in general agreement with this fracture criterion. For quantitative agreement, finite extensibility effects have to be considered. (C) 2017 The Society of Rheology.

https://doi.org/10.1122/1.4995497
\end{abstract}

\section{INTRODUCTION}

The phenomenon of failure in elongational deformation of molten polymeric systems is among the least known and most researched fields in the study of soft matter. This phenomenon has been subjected to vast experimental, theoretical, and computational research endeavors for a long time, while the portrayal of a clear picture of the failure mechanism is yet to be accomplished. The occurrence of failure, which includes necking ("ductile failure") and rupture or brittle fracture ("cohesive failure") during extensional flow, is of significant concern for the polymer industry when processes such as blow molding, fiber spinning, film blowing, and paint spray are involved. Moreover, the defects in the extrusion process such as melt fracture and sharkskin are directly linked to this phenomenon. The understanding of failure requires a systematic knowledge of the nonlinear rheology of polymer melts, and its correlation with molecular aspects such as entanglements. The nonlinear deformation behavior of entangled polymers is mainly dependent on the response of the topological constraints resulting from the intertwining of polymer chains to the imposed deformation. Due to the substantial dependence of extensional deformations on the molecular structure of the polymer (degree of branching, molecular weight, polydispersity, etc.), the understanding of this type of flow not only sheds light on the mechanism of failure but also reveals the role of entanglements and other molecular characteristics

\footnotetext{
a) Author to whom correspondence should be addressed; electronic mail: manfred.wagner@tu-berlin.de

(C) 2017 by The Society of Rheology, Inc.

behind this phenomenon. However, due to the clear limitation of reliable datasets on failure, the quantitative modeling of this phenomenon to predict its occurrence, as well as the understanding of its governing molecular mechanisms, has remained an elusive goal so far [1,2].

Reiner and Freudenthal [3] proposed a dynamical theory of strength based on the assumption that tensile stress exceeding a critical value $(6 K G)^{1 / 2}$ will lead to rupture, where $G$ is the shear modulus and $K$ is the elastic energy per unit volume (i.e., resilience). The Reiner theory states that ruptures occur at a critical strain that is independent of the stretch rate and demonstrates a strain-rate independence for the failure strain in the rubbery regime and a stress at rupture that is rate independent [2].

The Considère construction [4] is a concept from solid mechanics and states that the nonuniform deformation (necking) occurs when the engineering stress (tensile force/ initial cross sectional area) passes through a maximum. The mentioned maximum in the Considère criterion sets the limiting strain for uniform extension. McKinley and Hassager [5] applied the Doi-Edwards (DE) [6] and pompom [7] models in conjunction with the Considère criterion to simulate the melt behavior of linear and long-chain branched (LCB) polymers, respectively. According to this construction, uniform uniaxial extensional deformation cannot occur after the force maximum (corresponding to low strain); yet, this statement is in clear contradiction with the extensional experimental studies. This construction at best provides a good estimate of necking at high Weissenberg numbers, although the failure in this region is mainly due to rupture. 
Vinogradov and coworkers were first to develop an extensive body of data on failure and rupture in the extensional flow of monodisperse polymer melts [8-14]. Later, Malkin and Petrie [15] presented a master curve based on the data of Vinogradov et al. describing different regimes of failure as a function of strain rate. Malkin and Petrie's work [15] demonstrates four zones of failure during extensional flow: Steadystate elongational flow can be observed at very low rates only (purely viscous zone), while necking instabilities take place at higher elongation rates (viscoelastic zone). Necking ultimately leads to failure, which prevents steady flow. This process is a fluid mechanical phenomenon distinct from solidlike fracture. At high (rubbery zone) and very high (glassy zone) elongation rates, the polymer fractures in a rubber- or solidlike fashion.

In a series of studies [16-20], Wang and coworkers concluded that the maximum in engineering tensile stress $\sigma_{\text {eng }}$ coincides with the onset of necking. Hence, they concluded that the maxima in the tensile force in extensional deformation have the same mechanism as those observed in shear. According to the authors, the visible material failure due to nonuniform extension representing the yield point (i.e., the maximum of $\sigma_{\text {eng }}$ ) occurs when the elastic retraction force equals the intermolecular gripping force, and the molecular deformation ceases to increase (and eventually starts decreasing) due to interchain sliding, leading to disentanglement. However, beyond a critical strain rate, entangled melts and solutions were found to undergo a yield-to-rupture transition [18-20]. Wang and coworkers showed that the onset of the "glasslike" zone as defined by Malkin and Petrie [15] is actually in the middle of the rubbery plateau where the mechanical response of entangled melts is not dictated by glassy chain dynamics. Birefringence measurements indicate that rupture takes place when the strands between entanglements undergo non-Gaussian stretching on their path to full extension, and the linear stress-optical rule starts to break down. The authors concluded that rupture occurs plausibly through chain scission in the limit of finite chain extensibility.

Progress on the experimental side has recently been made by the seminal work of Huang et al. [21] and Huang and Hassager [22] on well characterized monodisperse polystyrenes (PSs) dispersed in oligomeric styrene (OS) via VADER 1000, Rheo Filament ApS, a commercially available filament stretching rheometer [23]. By measuring the local diameter of the polymer sample during elongation, the true Hencky strain and strain rate can be determined, while from the global deformation of the sample, only nominal values of strain and strain rate can be obtained. Huang and Hassager showed [22] that when true Hencky strain rates are utilized rather than nominal Hencky rates, the four zones in the Malkin plot are reduced to just two states: liquid and solid, and a clear distinction exists between liquid (steady-state elongation) behavior and solid (fracture) behavior. For two monodisperse PS solutions (with $33 \%$ and $17 \%$ polymer volume fractions), they found that the value of the critical stress for rupture is between the plateau $\left(G_{e}\right)$ modulus and the glassy $\left(G_{0}\right)$ modulus, and at least 2 orders of magnitude higher than $G_{e}$. The authors confirmed that within $4 \leq \mathrm{Wi}_{R} \leq 100$, the critical strain remains constant and the critical stress increases only slightly with increasing elongation rate.

Based on Vinogradov and coworker's dataset [8-14], several research groups attempted to analyze and rheologically model the failure phenomenon in melts [2,5,16-20,24-27]. However, those modeling attempts were based on the conceptual and schematic failure master curve produced by Malkin and Petrie [15] and derived from elongational measurements based on nominal Hencky strain rates, which was recently challenged by the Filament Stretching Rheometer's ability to measure the true Hencky rate [21,22] as discussed earlier. The scaling theory of Joshi and Denn [2,24,25] demonstrated impressive qualitative agreement with the narrowdistribution polyisoprene failure data of Vinogradov's team within about $50 \%$ uncertainty in the reptation time [2]. This theory is based on the scaling analysis of Brochard and de Gennes [28] for failure in shear ("slip") near a solid surface. It assumes that catastrophic failure occurs when the frictional force of an entangled chain can no longer balance the tension in the molecular chain and predicts failure by disentanglement of the polymer chains considering the recoverable proportion of the total strain. It should be noted that the failure model of Joshi and Denn [2,24,25] predicts an increasing strain to failure with increasing Weissenberg number in the viscoelastic and the rubbery failure zone, and is therefore not in agreement with the experimental data of Huang et al. [21] and Huang and Hassager [22] for well characterized monodisperse PS solutions featuring a constant critical strain within the experimental range of $4 \leq \mathrm{Wi}_{R} \leq 100$.

The origin of crack initiation in polymeric solutions and melts has not been broadly studied. The idea of the largest pre-existing microcrack (i.e., weakest-link), inherited from the Griffith theory [29], is not in agreement with the data of Huang and Hassager, owing to the extreme reproducibility of critical stress/strain, as well as simultaneous initiation and propagation of multiple cracks at different spatial positions in polymer solutions as observed in $[21,22]$. To account for the incongruities between the Griffith theory and the experimental observations during the uniaxial deformation of polymer solutions, Huang et al. [21] proposed a thermally induced fluctuation mechanism for crack initiation in melts and solutions based on a modified Griffith theory [29] developed by Pomeau [30] and used by Tabuteau and coworkers [31,32] for oil-in-water droplet microemulsion connected to each other by telechelic polymers. They hypothesized that thermal fluctuations result in local spots of lower polymer density. However, it should be noted that this model does not take into account the presence of entanglements (see, e.g., review by Ligoure and Mora [33]), and the fracture in the systems studied by Tabuteau et al. occurs already in the linear-viscoelastic regime, which is clearly not the case for the entangled polymer solutions studied by Huang et al. [21,22].

As entangled polymer solutions and melts fracture in the rubbery zone, i.e., at elongation rates, which are larger than the inverse Rouse time $\tau_{R}$ of the polymer chain, it may be useful to consider shortly the fracture of crosslinked rubber networks. Fracture of rubbers causes inevitably the breakage of covalent bonds. Lake and coworkers [34-36] assumed that when a network strand with $N_{x}$ carbon bonds between 
two chemical crosslinks is stretched and fails by rupture of one $\mathrm{C}-\mathrm{C}$ bond along the strand, an energy equivalent to $N_{x}$ times the bond energy $U$ of one $\mathrm{C}-\mathrm{C}$ bond is dissipated, because all carbon bonds in the strand are stretched to the limit. Therefore, the threshold fracture energy $\Gamma_{0}$ per unit of fracture surface area is given by

$$
\Gamma_{0} \cong N_{x} U \Sigma
$$

with

$$
\Sigma \cong \nu l N_{x}^{1 / 2}
$$

where $\Sigma$ is the areal density of chains crossing the interface, $U$ the $\mathrm{C}-\mathrm{C}$ bond energy, $\nu$ the density of network strands, and $l$ the length of a monomer unit. With $\rho$ and $M_{0}$ being the monomeric density and molar mass, this results in

$$
\Gamma_{0} \cong U \frac{l \rho}{M_{0}} N_{x}^{1 / 2}
$$

The threshold fracture energy $\Gamma_{0}$ can be measured directly in tearing tests by extrapolating the energy required to create a unit of facture surface to zero crack speed, thereby eliminating other dissipative processes [37]. Equation (3) is in qualitative agreement with data of elastomers [37] and hydrogels [38]. However, Mazich and Samus [39] found that for a pol$\mathrm{y}$ (dimethylsiloxane) (PDMS) network with a large number of trapped entanglements, the molar mass $M_{e}$ between entanglements determines the threshold fracture energy and showed that $\Gamma_{0} \propto\left(M_{e} / M_{0}\right)^{1 / 2}$, i.e., the threshold fracture energy depends on the number of $\mathrm{C}-\mathrm{C}$ bonds in an entanglement segment.

It should be noted that the threshold fracture energy cannot be determined in a tensile test, because the nonaffine deformation of the network strands is not known a priori, and the crack speed (and therefore other dissipative processes than fracture) cannot be controlled. What can be determined is the critical Hencky strain at rupture, $\varepsilon_{c}$, and the critical stress at rupture, $\sigma_{c}$, which is related to the rupture force $F_{c}$ of a $\mathrm{C}-\mathrm{C}$ bond by $\sigma_{c} \cong \nu F_{c} L$, where $\nu$ is the density and $L$ the length of the fully stretched network strands. From atomic force microscope experiments, the rupture force is reported as $F_{c} \cong 4 \mathrm{nN}=4 \times 10^{-9} \mathrm{~N}$ [40]. $A b$ initio calculations predict rupture forces of $6.8 \mathrm{nN}$ for polyisoprene and $7.2 \mathrm{nN}$ for polybutadiene [41].

Inspired by the findings of Mazich and Samus [39] that trapped entanglements determine the fracture of polymer networks, we consider in this contribution the brittle fracture of entangled polymer solutions and melts in elongational flow and present a novel hypothesis for crack initiation based on energy considerations. Model predictions are then compared to the experimental data of Huang and coworkers [21,22,42-45].

\section{THE STRAIN ENERGY OF A CHAIN IN A TUBE}

Starting from the tube model, the entropic free-energy $w_{s}$ of a chain segment of a linear entangled chain with a large number of monomers $N_{e}$ (Kuhn steps with length $b$ ) in a tube with diameter $a$ is given by [46-48]

$$
w_{s}=3 k T \frac{N_{e} b^{2}}{a^{2}}+c s t .
$$

If $N_{e}$ represents the number of monomers in an entanglement segment of length and diameter $a_{0}$ at equilibrium with $N_{e} b^{2}=a_{o}^{2}$, and the constant cst is set to zero, $w_{s}$ represents the free energy of an entanglement segment confined in a tube with strain-dependent tube diameter $a$,

$$
w_{s}=3 k T \frac{a_{0}^{2}}{a^{2}}=3 k T f^{2} .
$$

$f$ has been called the molecular stress function (MSF) [49]. In the Gaussian regime, stress is proportional to stretch, and $f$ also represents the molecular stretch. The thermal energy $w_{\text {eq }}$ of the entanglement segment at equilibrium, i.e., when $a=a_{0}$ and $f=1$, is then simply given by

$$
w_{\mathrm{eq}}=3 k T \text {. }
$$

At larger deformations, i.e., smaller tube diameters $a$, the strain energy of the entanglement segment is a multiple of $w_{\text {eq }}$,

$$
w_{s}=w_{\mathrm{eq}} f^{2},
$$

i.e., the strain energy increases with the square of chain stretch $f$, as long as Gaussian chain statistics apply and finite extensibility effects can be neglected.

\section{THE BOND ENERGY OF CARBON-CARBON BONDS}

The bond energy of carbon-carbon bonds in hydrocarbons is reported as $348 \mathrm{~kJ} / \mathrm{mol}$ (see, e.g., [36,37]), i.e., the bond energy of a single $\mathrm{C}-\mathrm{C}$ bond is with Avogadro's number $N_{A}=6.02 \times 10^{23}$

$$
U=\frac{348 \mathrm{~kJ}}{N_{A}}=5.78 \times 10^{-19} \mathrm{~J} .
$$

On the other hand, the thermal energy $w_{\text {eq }}$ at a temperature $T$ of $393 \mathrm{~K}\left(120^{\circ} \mathrm{C}\right)$ is

$$
w_{\text {eq }}=3 k T=1.62 \times 10^{-20} \mathrm{~J},
$$

with Boltzmann's constant $k=1.38 \times 10^{-23} \mathrm{~J} / \mathrm{K}$. Thus, the bond energy $U$ is 36 times larger than the thermal energy $w_{\text {eq }}$, which is why the polymer chain will not rupture due to Brownian motion at equilibrium, while chain scission may be caused by ultraviolet radiation with more than $3.6 \mathrm{eV}$ [40].

\section{HYPOTHESIS ON FRACTURE INITIATION}

The strain energy of an entanglement segment is distributed along the segment. However, we make the hypothesis 
that when due to stretch, the energy of the entanglement segment reaches the critical energy

$$
w_{c}=3 k T f_{c}^{2}=U
$$

the total strain energy of the chain segment will be concentrated on one $\mathrm{C}-\mathrm{C}$ bond by thermal fluctuations, and this bond then ruptures. Thermal vibration frequencies are reported to be in the range of $\omega_{\mathrm{th}}=10^{10}-10^{12} \mathrm{~s}^{-1}$ [33] and include bond rotation and bond stretching. Using simplistically an Arrhenius ansatz, the lifetime of a $\mathrm{C}-\mathrm{C}$ bond can be estimated as $\tau_{0}=\left(1 / \omega_{\text {th }}\right) \exp (U / k T)$. According to the Eyring kinetic theory of fracture, the lifetime also depends on the force $F$ carried by a link of length $\delta$, whose stored energy can supply a portion of the activation energy [33]. If we identify this link with an entanglement segment carrying a strain energy $w_{s}$, the bond life time becomes $\tau=1 / \omega_{t h} \exp$ $\left(U-w_{s} / k T\right)=\tau_{0} \exp \left(-w_{s} / k T\right)$. When the strain energy $w_{s}$ reaches the bond energy $U$, the bond will rupture within a very short time. We assume that this will happen independently of the number of bonds, i.e., the length of the chain segment, as long as $N_{e}$ is large enough for Gaussian chain statistics to apply. When the bond ruptures, the newly created ends of the chain segment will recoil within a segmental Rouse time, thereby dissipating the strain energy, which may then be transmitted to primary $\mathrm{C}-\mathrm{C}$ bonds of adjacent chains leading to further chain scissions. When a sufficient concentration of locally ruptured chains is reached, crack initiation will occur. This can take place at several locations simultaneously along the polymer sample under tension, and highspeed images revealed indeed that multiple cracks propagate [21,22], one of which leading to rupture of the sample, while the others close up elastically. Cracks start always at the sample surface [21,22], which may be due to the specific condition at the polymer liquid/air interface being slightly different from the bulk. The surface energy that must be overcome for the creation of a critical crack size is extremely low, as the crack will start in the solvent of the polymer solution. Crack initiation is expected as soon as chains rupture, which leads within a very short time (about $200 \mathrm{~ms}$ according to Huang et al. [21]) to brittle fracture of the polymer liquid.

From the fracture hypothesis, Eq. (10), it follows with Eq. (7) a maximum achievable (critical) molecular stretch $f_{c}$

$$
f_{c} \leq \sqrt{\frac{U}{w_{\mathrm{eq}}}}=\sqrt{\frac{U}{3 k T}} \cong 6,
$$

at which rupture of primary $\mathrm{C}-\mathrm{C}$ bonds and thus brittle fracture is expected to occur. We call this fracture mode "entropic fracture," as it is caused by thermal fluctuations, in contrast to the enthalpic fracture hypothesis of Lake and Thomas [36] as modified by Mazich and Samus [39], assuming that when a chain with $N \mathrm{C}-\mathrm{C}$ bonds between two entanglements ruptures, the strain energy $w_{c}=N U$ corresponding to the bond energy of all $N \mathrm{C}-\mathrm{C}$ bonds in the entangled chain segment is dissipated. According to this assumption, the entangled chain will only rupture, if the entanglement segment is fully stretched to length $L \cong N l \cong N_{e} b$, i.e., when $f_{c} \cong N_{e}^{1 / 2}$, and when the covalent bonds are stretched by the tension in the chain to such an extent that the rupture force $F_{c}$ of the $\mathrm{C}-\mathrm{C}$ bonds is reached.

Next, we briefly recall a rheological constitutive equation which has been shown to accurately model the rheology of monodisperse polymer melts and solutions, and to allow the prediction of the molecular stretch $f$.

\section{THE MSF MODEL AND THE INTERCHAIN TUBE PRESSURE}

The MSF model is a generalized tube segment model with strain-dependent tube diameter [50-52]. According to this model, the segmental chain stretch, $f$, is inversely proportional to the diameter $a$ of a tube segment. The tube diameter can be viewed as the mean field of the surrounding chains which is independent of the orientation of tube segments, and it decreases from its equilibrium value $a_{0}$ with increasing stretch. The extra stress tensor $\sigma$ of the MSF model is expressed as

$$
\sigma(t)=\int_{-\infty}^{t} \frac{\partial G\left(t-t^{\prime}\right)}{\partial t^{\prime}} f^{2}\left(t, t^{\prime}\right) \mathbf{S}_{\mathrm{DE}}^{\mathrm{IA}}\left(t, t^{\prime}\right) d t^{\prime}
$$

Here, $G(t)$ is the linear-viscoelastic relaxation modulus, which may be expressed by a sum of discrete relaxation modes, $G(t)=\sum_{i=1}^{n} g_{i} e^{-t / \lambda_{i}} . \mathbf{S}_{\mathrm{DE}}^{\mathrm{IA}}$ is the DE orientation tensor assuming an independent alignment (IA) of tube segments [53], which is five times the second-order orientation tensor $\mathbf{S}$

$$
\mathbf{S}_{\mathrm{DE}}^{\mathrm{IA}}\left(t, t^{\prime}\right) \equiv 5\left\langle\frac{\mathbf{u}^{\prime} \mathbf{u}^{\prime}}{\mathbf{u}^{\prime 2}}\right\rangle=5 \mathbf{S}\left(t, t^{\prime}\right)
$$

$\mathbf{S}$ is the relative second-order orientation tensor. The bracket denotes an average over an isotropic distribution of unit vectors $\mathbf{u}\left(t^{\prime}\right)$ at time $t^{\prime}$ and can be expressed as a surface integral over the unit sphere

$$
\langle\rangle_{\mathrm{o}} \equiv \frac{1}{4 \pi} \oiint[] \sin \theta d_{o} \theta_{o} d \varphi_{o} .
$$

At the observation time $t$, the unit vectors are deformed to vectors $\mathbf{u}^{\prime}$, which are calculated from the affine deformation hypothesis [with $\mathbf{F}^{-1}\left(t, t^{\prime}\right)$ as the relative deformation gradient tensor] as

$$
\mathbf{u}^{\prime}\left(t, t^{\prime}\right)=\mathbf{F}^{-1}\left(t, t^{\prime}\right) \cdot \mathbf{u}\left(t^{\prime}\right)
$$

$u^{\prime}$ indicates the length of the vector $\mathbf{u}^{\prime}$.

The MSF $f=f\left(t, t^{\prime}\right)$ is the inverse of the relative tube diameter

$$
f\left(t, t^{\prime}\right)=a_{0} / a\left(t, t^{\prime}\right)
$$

$f=f\left(t, t^{\prime}\right)$ is a function of both the observation time $t$ and the time $t^{\prime}$ of creation of tube segments by reptation. Thus, chain segments with long relaxation times, i.e., those 
preferably in the middle of the tube, are exposed to higher stretches than chain segments with short relaxation times, i.e., those at the chain ends.

Assuming an on average affine deformation of the chain, the evolution equation for $f$ can be expressed as

$$
\frac{\partial f}{\partial t}=f(\mathbf{K}: \mathbf{S})
$$

with the starting condition $f\left(t=t^{\prime}, t^{\prime}\right)=1$, which has the solution [49]

$$
f=\exp \left(\left\langle\ln \left(u^{\prime}\right)\right\rangle\right)=\exp \left(\frac{w_{\text {or }}}{3 k T}\right)
$$

$w_{\text {or }}$ is the orientational free energy of a tube segment and represents the strain energy potential of the DE IA stress tensor [Eq. (13)]. For elongational flows, $w_{\text {or }}$ can be expressed in analytical form in terms of Hencky strain $\varepsilon$ by the use of Eqs. (14) and (15) as

$$
\frac{w_{o r}}{3 k T}=\frac{1}{\sqrt{\exp (3 \varepsilon)-1}} \tan ^{-1}(\sqrt{\exp (3 \varepsilon)-1})+\varepsilon-1 .
$$

In the elastic limit, i.e., without tube diameter relaxation effects, Eq. (18) relates molecular stretch $f$ as well as the free energy $w_{s}$ of a tube segment [Eq. (5)] directly to macroscopic deformation of the sample, and the extra stress [Eq. (12)] can be derived from the strain energy potential $W=\nu w_{s}[51]$.

Considering stretch relaxation processes, Pearson et al. [54] assumed that the stretch created by affine deformation of the chain is balanced by a linear spring force. The corresponding evolution equation of the MSF is then expressed as

$$
\frac{\partial f}{\partial t}=f(\mathbf{K}: \mathbf{S})-\frac{1}{\tau_{R}}(f-1)
$$

where $\tau_{R}$ is the Rouse stretch-relaxation time of the chain, and $\mathbf{K}$ the velocity gradient tensor. However, in elongational flow with the strain rate $\dot{\varepsilon}$, Eq. (19) leads to unbounded stretch at Weissenberg numbers $W i=\dot{\varepsilon} \tau_{R} \rightarrow 1$, and is not in agreement with the steady-state elongational viscosity data of monodisperse PS melts [55,56], which show a continuously decreasing elongational viscosity with a scaling of approximately $(\dot{\varepsilon})^{-1 / 2}$. To explain this scaling, Marrucci and
Ianniruberto [57] proposed an interchain pressure (IP) term based on the free energy of a polymer chain confined by fixed walls of a tube [53]. They assumed that the reduction of the tube diameter due to deformation is balanced by an increase of the radial pressure of the confined chain. Later, by considering that the pressures of a chain exerted on the walls of a confining box are anisotropic if the dimensions of the box are anisotropic, an extended interchain pressure (EIP) model was developed with $1 / 3$ Rouse relaxation in the longitudinal direction (no walls) and $2 / 3$ IP in the lateral direction of a tube segment [58]

$$
\frac{\partial f}{\partial t}=f(\mathbf{K}: \mathbf{S})-\frac{1 f-1}{3} \frac{2 f^{2}\left(f^{3}-1\right)}{3 \tau_{R}} .
$$

Equation (20) becomes equivalent to Eq. (19) in the first order of stretch, i.e., for $f-1 \ll 1$, but avoids the singularity in the classical relation when $\mathrm{Wi} \rightarrow 1$. It does not contain any nonlinear parameter since the Rouse time of the chain can be evaluated from linear viscoelasticity. Thus, Eqs. (12) and (20) represent a nonlinear integro-differential constitutive relation with no free parameters. This was shown to be in excellent agreement with start-up and steady-state elongational viscosity data of several linear monodisperse PS melts [58], and of concentrated solutions of monodisperse PS in OS [59]. These findings demonstrated the importance of the IP term in polymer rheology.

For solutions, the shift $a_{T g}$ of the glass transitions temperature from melt to solution has to be taken into account and the evolution equations is given by [60]

$$
\frac{\partial f}{\partial t}=f(\mathbf{K}: \mathbf{S})-\left(1-\frac{2}{3} a_{T g}\right) \frac{f-1}{\tau_{R}}-\frac{2}{3} a_{T g} \frac{f^{2}\left(f^{3}-1\right)}{3 \tau_{R}} .
$$

\section{COMPARISON OF THE NOVEL FRACTURE HYPOTHESIS TO EXPERIMENTAL EVIDENCE}

Here, we compare predictions of our entropic fracture model for the origin of brittle fracture of entangled polymer liquids to the experimental data of Huang and coworkers. Table I gives the samples name, molecular weight, polydis-

\begin{tabular}{|c|c|c|c|c|c|c|}
\hline Sample name & $M_{w}$ of PS $(\mathrm{kg} / \mathrm{mol})$ & PDI & Oligomeric styrene $(\mathrm{kg} / \mathrm{mol})$ & PS (wt. \%) & Test or reference temp. $\left({ }^{\circ} \mathrm{C}\right)$ & Study \\
\hline PS-864k/4k-17 & 864 & $<1.15$ & 4 & 17 & 120 & {$[21,22]$} \\
\hline PS-864k/4k-33 & 864 & $<1.15$ & 4 & 33 & 120 & {$[21,22]$} \\
\hline PS-545 k/1 k-52 & 545 & 1.12 & 1 & 52 & 130 & {$[44,59]$} \\
\hline PS-545 k/2 k-58 & 545 & 1.12 & 2 & 58 & 130 & {$[59,61]$} \\
\hline PS-545 k/4k-17 & 545 & 1.12 & 4 & 17 & 120 & {$[21]$} \\
\hline PS-900k/4k-33 & 900 & 1.10 & 4 & 33 & 130 & {$[45]$} \\
\hline PS-3280k/4 k-13 & 3280 & 1.11 & 4 & 13 & 130 & {$[45]$} \\
\hline PS-CAS-0993-53-6 & 230 & 3.7 & - & 100 & 120 & {$[21,42]$} \\
\hline
\end{tabular}
persity index, weight fraction, and testing or reference temperature of 7 solutions of PS in OS as solvent, and one PS melt investigated in uniaxial extensional deformation tests via filament stretching rheometry.

TABLE I. Samples description of PS solutions and melt. 
Figure 1 shows the experimental data of true stress as a function of Hencky strain for PS-864k/4k-17 and PS-864k/ 4k-33. Dotted lines indicate predictions of Eqs. (12) and (18), which are in excellent agreement with experimental data up to a Hencky strain of 2, when the stress data show a stronger increase than predicted. Solid lines are predictions of Eqs. (12) and (18) assuming that the entanglement stretch is limited to $f_{c}=6$. As demonstrated in Fig. 1, the entropic rupture hypothesis, Eq. (11), is in general agreement with the Hencky strain at rupture observed.

Employing evolution equation (21) instead of Eq. (18), i.e., allowing for tube diameter relaxation, has little effect on the predictions, as the Weissenberg number Wi $\gg 1$ (Fig. 2). The maximal value of the MSF follows from Eq. (21) at $\partial f / \partial t$ $=0$ and can be approximated for sufficiently large $f$ by

$$
f_{\max }^{2} \cong \frac{3}{2} \sqrt{2 \frac{\dot{\varepsilon} \tau_{R}}{a_{T g}}}=\frac{3}{2} \sqrt{2 \mathrm{Wi}} .
$$

According to the hypothesis proposed, rupture will occur at strain rates, for which $f_{\max } \geq f_{c}=6$, i.e., for $\mathrm{Wi}=\left(\dot{\varepsilon} \tau_{R} / a_{T g}\right)$ $\geq 288$. For PS-864/4k-33, the limiting strain rate for rupture is predicted to be $0.0075 \mathrm{~s}^{-1}$, and for PS-864/4k-17 it is $0.012 \mathrm{~s}^{-1}$ (see in Fig. 3 the divergence of the strain at rupture predicted). Below these strain rates, we expect that steadystate elongation is possible. As can be seen from Fig. 3, the strain at rupture is correctly predicted by the entropic fracture hypothesis for PS-864/4k-17, while PS-884/4k-33 ruptures already at a smaller Hencky strain.

For sufficiently fast deformations, from Eqs. (12) and (22), the fracture stress is predicted to be

$$
\sigma_{c} \cong 5 G_{e} f_{c}^{2}=180 G_{e}=180 G_{N}^{0} \varphi^{2} .
$$

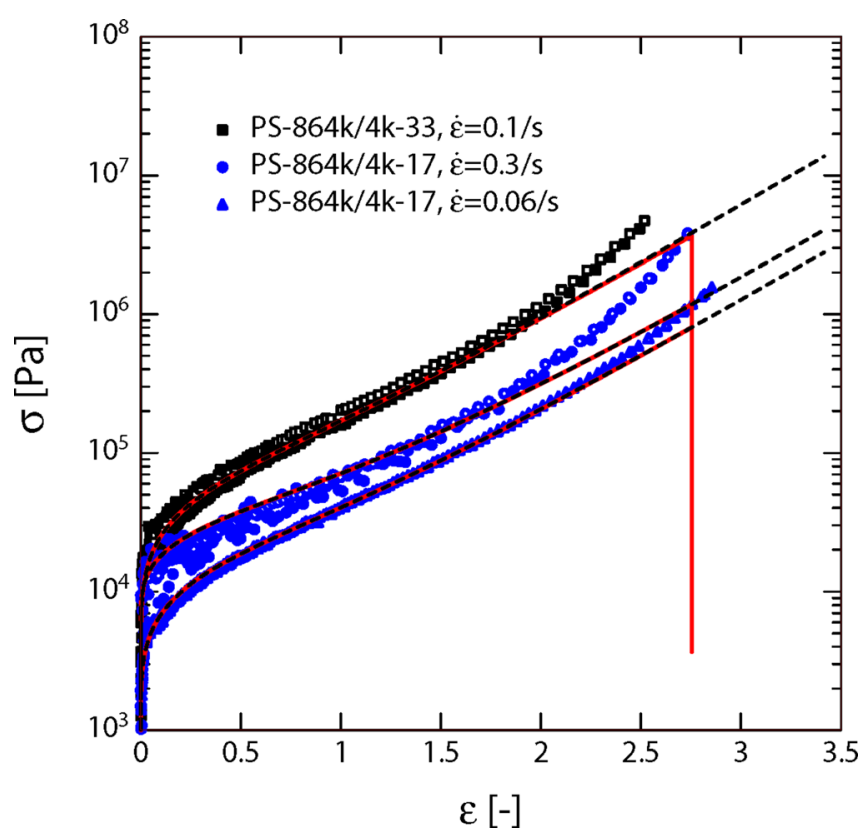

FIG. 1. Experimental data (symbols) of stress as a function of Hencky strain for PS-864k/4k-17 and PS-864k/4k-33. Open symbols indicate repeat experiments. Dotted lines: Predictions of Eqs. (12) and (18). Full lines: Predictions of rupture of PS solutions by Eqs. (12) and (18) with $f_{c}=6$.

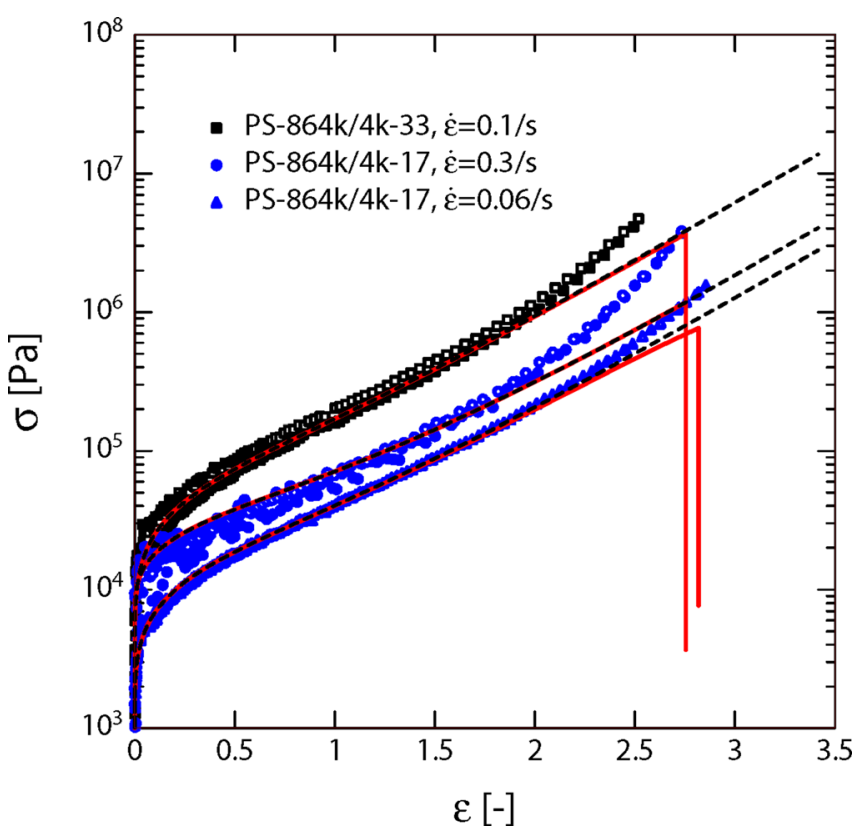

FIG. 2. Experimental data (symbols) of stress as a function of Hencky strain for PS-864k/4k-17 and PS-864k/4k-33. Dotted lines: Predictions of Eqs. (12) and (18). Full lines: Predictions of rupture of PS solutions by Eqs. (12) and (21) with $f_{c}=6$.

$G_{e}$ is the plateau modulus of the polymer solution considered, $G_{N}^{0}$ the plateau modulus of the melt (taken as $G_{N}^{0}=2 \times 10^{5} \mathrm{~Pa}$ for PS) and $\varphi$ the polymer volume fraction [the exponent of 2 in Eq. (23) assumes a dilution exponent of 1]. For PS-864/4k-33, Eq. (23) results in $\sigma_{c} \cong 180 G_{N}^{0} \varphi^{2}$ $=4 \times 10^{6} \mathrm{~Pa}$, which is in good agreement with the experimental data shown in Fig. 4, while for PS-864/4k-17, a value of $\sigma_{c} \cong 180 G_{N}^{0} \varphi^{2}=10^{6} \mathrm{~Pa}$ is predicted, which is somewhat

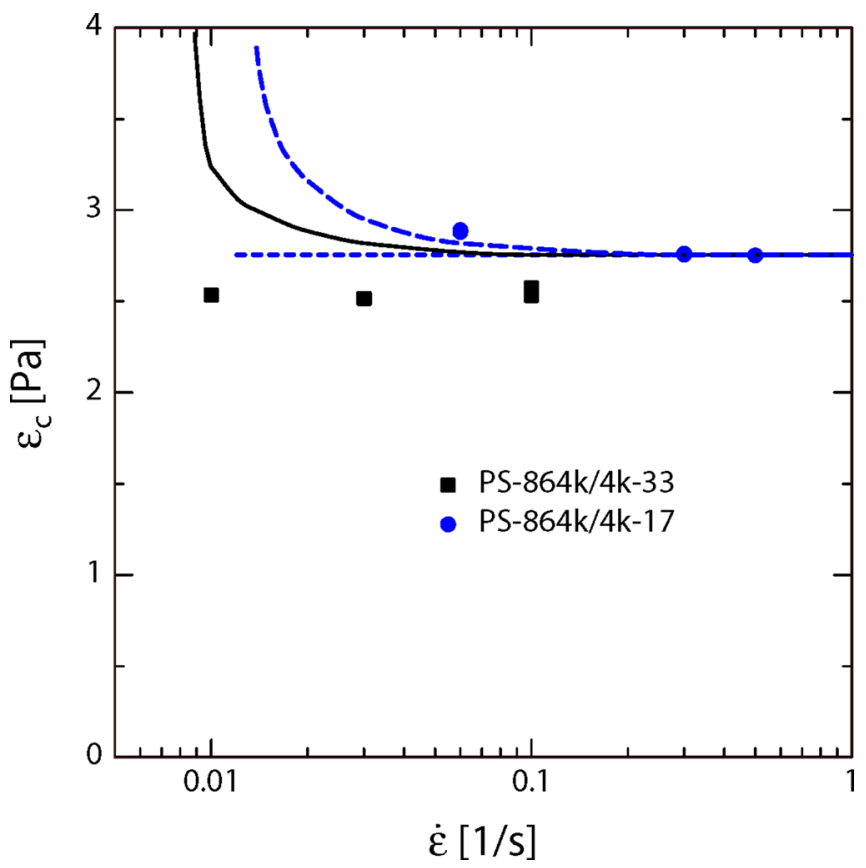

FIG. 3. Experimental data (symbols) of strain at rupture $\varepsilon_{c}$ of PS solutions as a function of strain rate. Predictions of Eqs. (12) and (21) with $f_{c}=6$ for PS-864/4k-33 (solid line) and PS-864/4k-17 (long dotted line). Dotted line indicates $\varepsilon_{\mathrm{c}}$ from Eqs. (12) and (18) with $f_{c}=6$. 


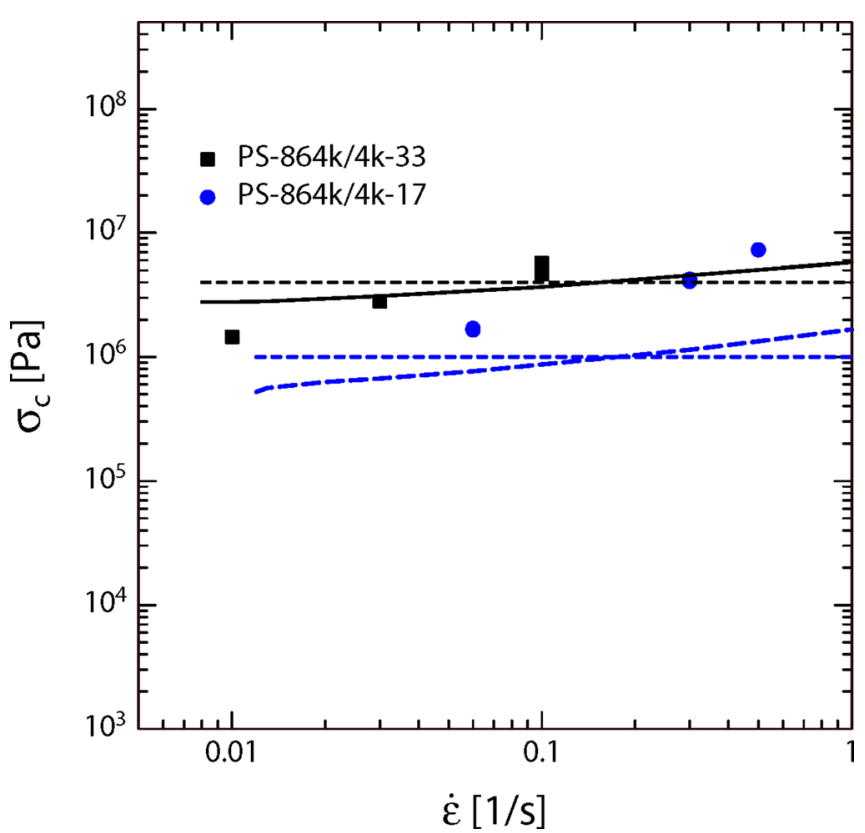

FIG. 4. Experimental data (symbols) of the stress at rupture $\sigma_{\mathrm{c}}$ of PS solutions as a function of strain rate. Predictions of Eqs. (12) and (21) with $f_{c}=6$ for PS-864/4k-33 (solid line) and PS-864/4k-17 (long-dotted line). Predictions of Eq. (23) are indicated by horizontal short-dotted lines.

smaller than the experimental data. The weak increase of the fracture stress with increasing strain rate as predicted by Eqs. (12) and (21) is due to the combined effect of tube diameter relaxation and contributions of the glassy relaxation modes of the polymer solutions.

While the entropic fracture model is in general (if not fully quantitative) agreement with the experimental data of PS-864/ $4 \mathrm{k}-33$ and PS-864/4k-17, predictions of the enthalpic fracture hypothesis do not agree with experimental evidence: According to Fang et al. [62], the maximum stretch $\lambda_{m}$ for PS melt can be obtained as $\lambda_{m}=d_{t} / b$, where $b=1.48 \mathrm{~nm}$ is the Kuhn step length for PS [63], and $d_{t}$ the average end-to-end distance of the entanglement segment. This is related to the packing length $p$ by $d_{t}=19 p$, with $p=0.3948 \mathrm{~nm}$ for PS [64,65], leading to a value of $\lambda_{m}=5.07$. Alternatively, $\lambda_{m}$ can be calculated from $\lambda_{m}=0.82 \sqrt{M_{e} / M_{b} / C_{\infty}}$ [66]. The factor 0.82 takes into account the arrangement of monomer units in the "zig-zag" conformation of the chain when $M_{e}$ is the molar mass per entanglement, $M_{b}$ is the molar mass of the monomer, and $C_{\infty}$ is the characteristic ratio. For PS with $M_{e}=18100 \mathrm{~g} \mathrm{~mol}^{-1}$ [67], $M_{b}=52 \mathrm{~g} \mathrm{~mol}^{-1}$ and $C_{\infty}$ $=9.64$ [63], a value of $\lambda_{m}=4.93$ is obtained. We use a value of $\lambda_{m}=5$ for PS melt in the following. For PS solutions, the maximum stretch is therefore $\lambda_{m} \cong 5 \varphi^{-1 / 2}$ (see e.g., [68], which results in $\lambda_{m} \cong 8.7$ for PS-864/4k-33, and $\lambda_{m} \cong 12.1$ for PS-864/4k-17. This is clearly much larger than the critical stretch observed, which means that entanglement segments at rupture are not fully stretched. Also, according to the enthalpic fracture hypothesis, the critical stress needed to rupture the chains (assuming fully stretched and oriented chain segments) would be

$$
\sigma_{c} \cong \nu F_{c} L \cong \frac{G_{N}^{0} \varphi^{2}}{k T} F_{c} b \lambda_{m}
$$

With $b=1.48 \mathrm{~nm}$ for PS and $F_{c}=4 \mathrm{nN}$, this results in $\sigma_{c}$ $\cong 2.7 \times 10^{8} \mathrm{~Pa}$ for PS-864/4k-33, and $\sigma_{c} \cong 9.9 \times 10^{7} \mathrm{~Pa}$ for PS-864/4k-17, which is more than an order of magnitude larger than the stress at rupture observed experimentally.

Figures 5-7 demonstrate predictions of the entropic fracture model [Eqs. (12) and (21) with $f_{\mathrm{c}}=6$ ) for the polymer solutions PS-545k/2k-58, PS-545k/1k-52, and PS-900k/4k33 , which were investigated earlier by Huang and coworkers. For PS-545k/2k-58 (Fig. 5), fracture at a strain rate of $0.45 \mathrm{~s}^{-1}$ is predicted, while at lower strain rates, steady-state elongation at large strain is expected in accordance with experimental evidence. For solution PS-545k/1k-52, the entropic fracture model predicts fracture at the two highest strain rates investigated, which is in general agreement with experimental evidence, although fracture occurs at lower Hencky strains than predicted (Fig. 6). The evidence of fracture at the strain rate of $3.73 \mathrm{~s}^{-1}$ is less clear. In the case of PS-900k/4k-33 (Fig. 7), the model predicts brittle fracture at strain rate $0.002 \mathrm{~s}^{-1}$ in good agreement with experimental data, while no fracture at strain rate $0.007 \mathrm{~s}^{-1}$ is observed experimentally. However, the transition to steady-state elongation at lower strain rates is predicted correctly. It should be noted that no free parameter is used in any of these predictions so far, as all material parameters are fully determined by linear-viscoelastic data and the entropic fracture hypothesis as defined in Eq. (11).

\section{NON-GAUSSIAN EFFECTS}

Overall, predictions of the entropic fracture model are in general agreement with experimental data. However, in some cases, it seems that the model overpredicts the critical strain, and in other cases underpredicts the stress at rupture. It seems that the chain becomes stiffer with increasing strain loading as seen in Figs. 1 and 2 indicating a transition to

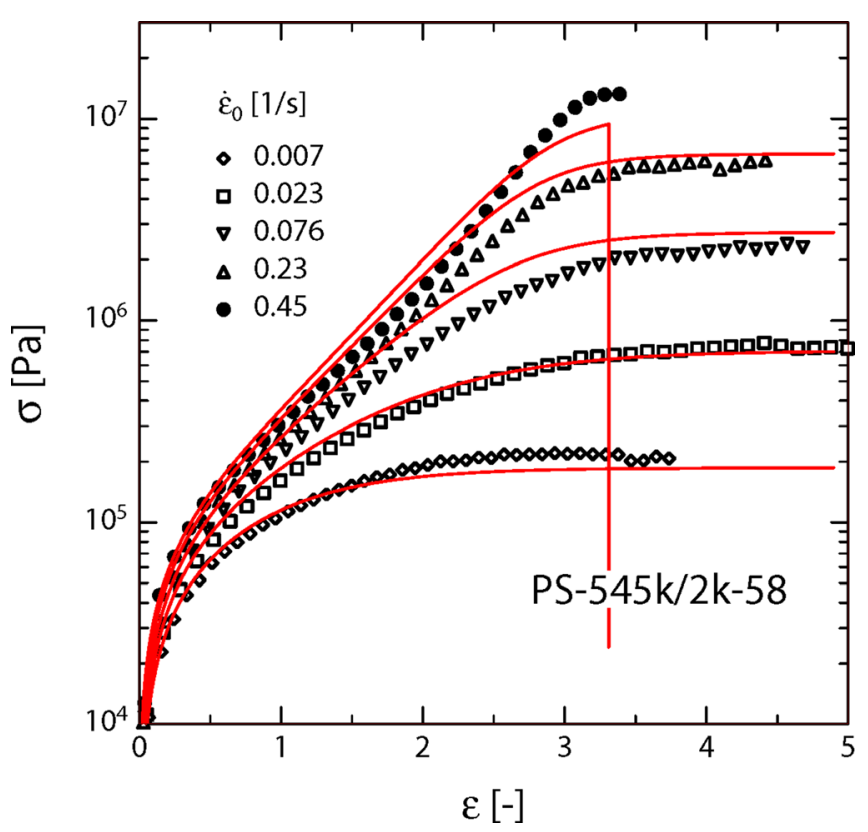

FIG. 5. Stress-strain data PS-545k/2k-58 (symbols). Predictions by Eqs. (12) and (21) limited to $f_{\mathrm{c}}=6$ (solid lines). 


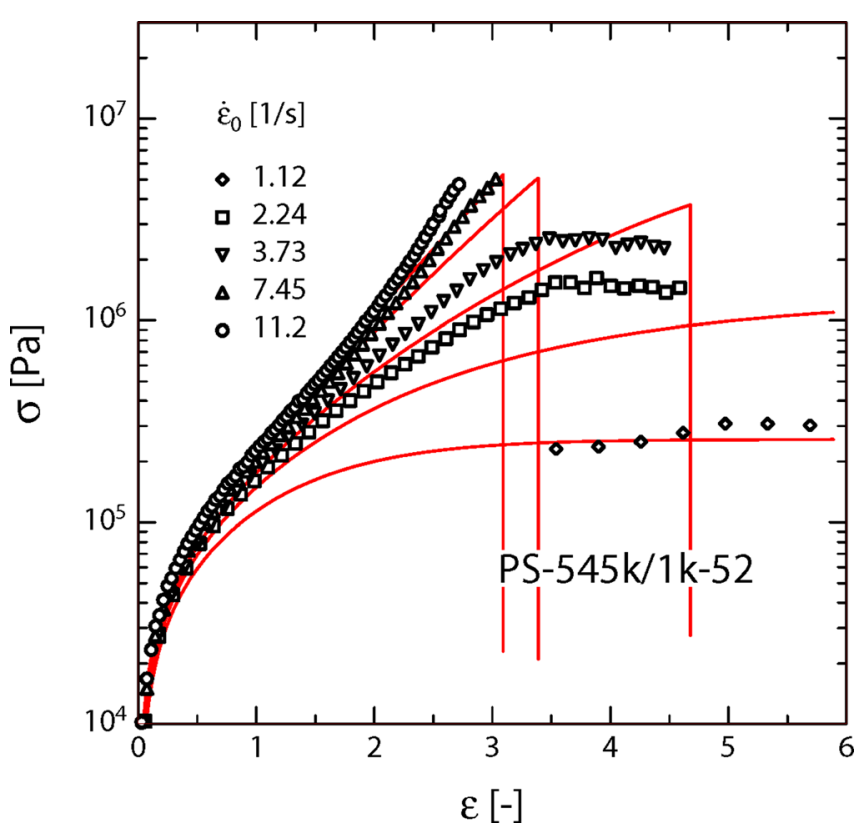

FIG. 6. Stress-strain data of PS-545k/1k-52 (symbols). Predictions by Eqs. (12) and (21) limited to $f_{\mathrm{c}}=6$ (solid lines).

non-Gaussian effects. This is in general agreement with the findings of Wang and coworkers [18-20] that rupture occurs when the strands between entanglements undergo nonGaussian stretching on their path to full extension, and the linear stress-optical rule starts to break down. According to Bird et al. [69], non-Gaussian effects become significant when the stretch reaches half of the maximum stretch $\left(f=0.5 \lambda_{\mathrm{m}}\right)$. In the following, we investigate possible consequences for the entropic fracture model.

Following Rolón-Garrido et al. [68], we model nonGaussian effects simplistically by introducing a stress enhancing factor $c\left(f^{2}\right)$ in the stress calculator

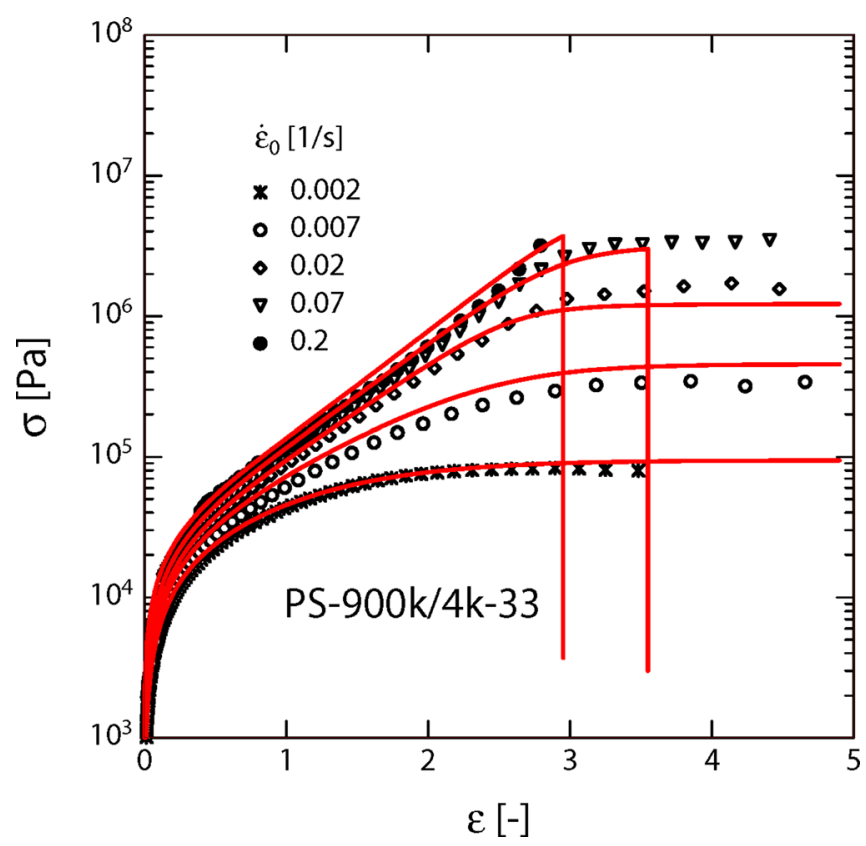

FIG. 7. Stress-strain data of PS-900k/4k-33 (symbols). Predictions by Eqs. (12) and (21) limited to $f_{\mathrm{c}}=6$ (solid lines).

$$
\sigma(t)=\int_{-\infty}^{t} \frac{\partial G\left(t-t^{\prime}\right)}{\partial t^{\prime}} c\left(f^{2}\right) f^{2}\left(t, t^{\prime}\right) \mathbf{S}_{D E}^{I A}\left(t, t^{\prime}\right) d t^{\prime}
$$

where $c$ is a nonlinear spring coefficient, representing a relative Padé inverse Langevin function with [70]

$$
c\left(f^{2}\right)=\frac{\left(3-\frac{f^{2}}{f_{m}^{2}}\right) \cdot\left(1-\frac{1}{f_{m}^{2}}\right)}{\left(3-\frac{1}{f_{m}^{2}}\right) \cdot\left(1-\frac{f^{2}}{f_{m}^{2}}\right)} .
$$

Reasonable agreement between the modified fracture model [Eqs. (21), (25), and (26)] and the data of the solutions PS864/4k-33, PS-864/4k-17, and PS-3280k/4k-13 is obtained for a value $f_{m}=6.5$ (Figs. 8-10). Interestingly, from the available experimental evidence, the finite extensibility parameter $f_{m}$ seems to be independent of the degree of dilution and thus from the maximal stretch $\lambda_{m}$, and is close to $f_{c}=6$, which means that stiffening of the chain may be caused by the reduction of the tube diameter, thus limiting the available lateral chain conformations and increasing nonentropic (enthalpic) effects. Also, as seen from Fig. 9, PS$864 / 4 \mathrm{k}-33$ ruptures already at $f_{c}=5$, possibly due to the lower number of monomers, $N_{e}=66.1$ [21], contained in an entanglement segment and lower maximal stretch $\lambda_{m} \cong 8.7$ compared to PS-864/4k-17 with $N_{e}=128.3$ [21] and $\lambda_{m} \cong 12.1$. Nearly quantitative agreement of the experimental data of strain (Fig. 9) and stress at rupture (Fig. 10) with predictions can be obtained for PS-864/4k-33 by use of $f_{c}=5$, while the stress at rupture of PS- $864 \mathrm{k} / 4 \mathrm{k}-17$ is in good agreement with the experimental data for $f_{c}=6$. For

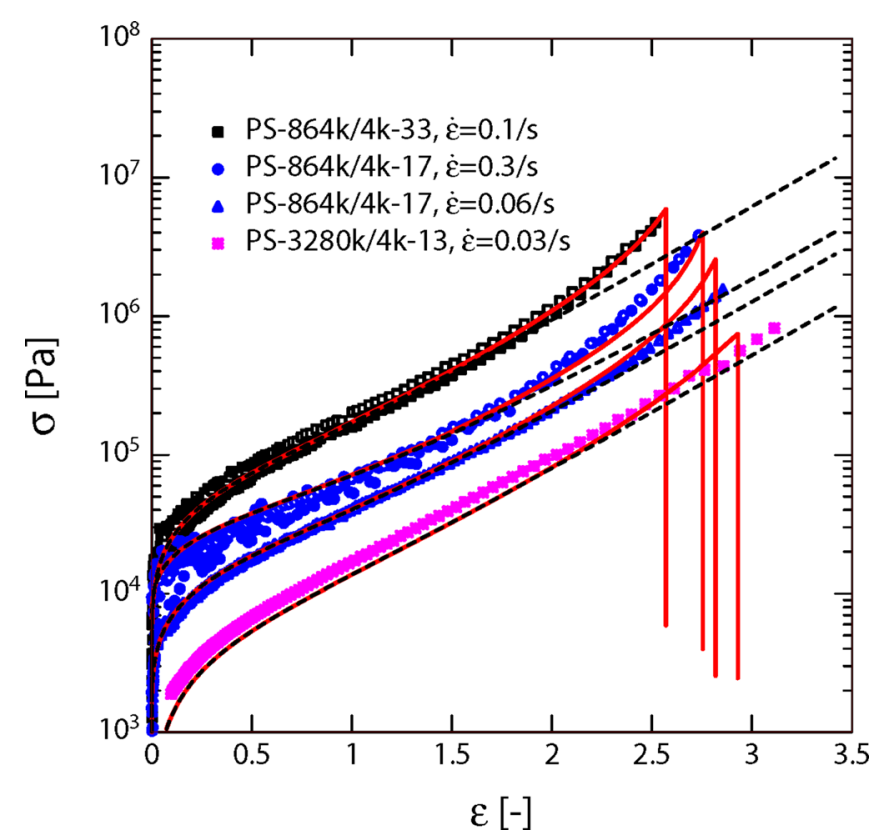

FIG. 8. Experimental data of stress as a function of Hencky strain for PS864k/4k-33, PS-864k/4k-17, and PS-3280k/4k-13 (symbols). Dotted lines: Predictions by Eqs. (12) and (18). Full lines: Predictions of rupture by Eqs. (21), (25), and (26) for PS-864/4k-33 ( $\left.f_{c}=5\right)$, and for PS-864/4k-17 and PS$3280 / 4 \mathrm{k}-13\left(f_{c}=6\right)$. Finite extensibility parameter $f_{m}=6.5$. 


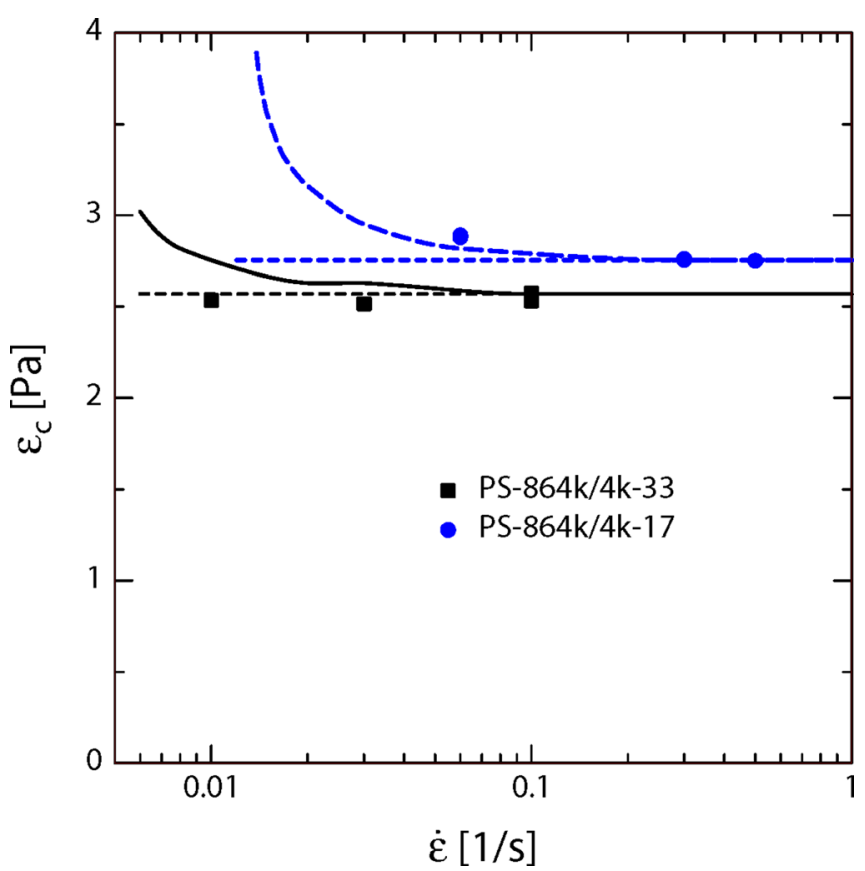

FIG. 9. Experimental data of strain at rupture $\varepsilon_{\mathrm{c}}$ of PS solutions as a function of strain rate (symbols). Predictions by Eqs. (12) and (18) with $f_{c}=5$ for PS$864 / 4 \mathrm{k}-33$ and $f_{c}=6$ for PS-864/4k-17 (short dotted lines). Predictions by Eqs. (21), (25), and (26) with $f_{c}=5$ for PS-864/4k-33, and $f_{c}=6$ for PS-864/ $4 \mathrm{k}-17$ (solid and long dotted lines, respectively). Finite extensibility parameter $f_{m}=6.5$.

the solution of PS-3280k/4k-13 with $N_{e}=169.2$ and maximal stretch $\lambda_{m} \cong 13.9$, the non-Gaussian effect seems to be small, as expected (Fig. 8).

For PS-864k/4k-17, the transition to steady elongational flow as predicted by Eq. (12) for strain rates less than $0.012 \mathrm{~s}^{-1}$, which is indicated by the divergence of the strain

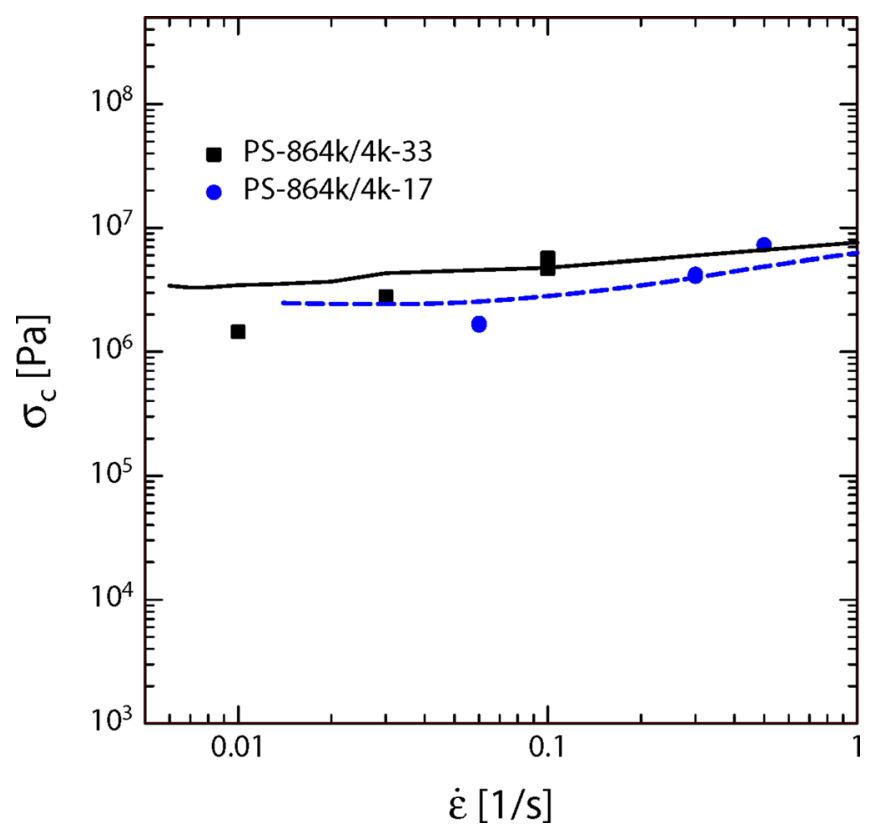

FIG. 10. Experimental data of the stress at rupture $\sigma_{\mathrm{c}}$ of PS solutions as a function of strain rate (symbols). Predictions by Eqs. (21), (25), and (26) with $f_{c}=5$ for PS-864/4k-33 (solid line), and $f_{c}=6$ for PS-864/4k-17 (dotted line). Finite extensibility parameter $f_{m}=6.5$.

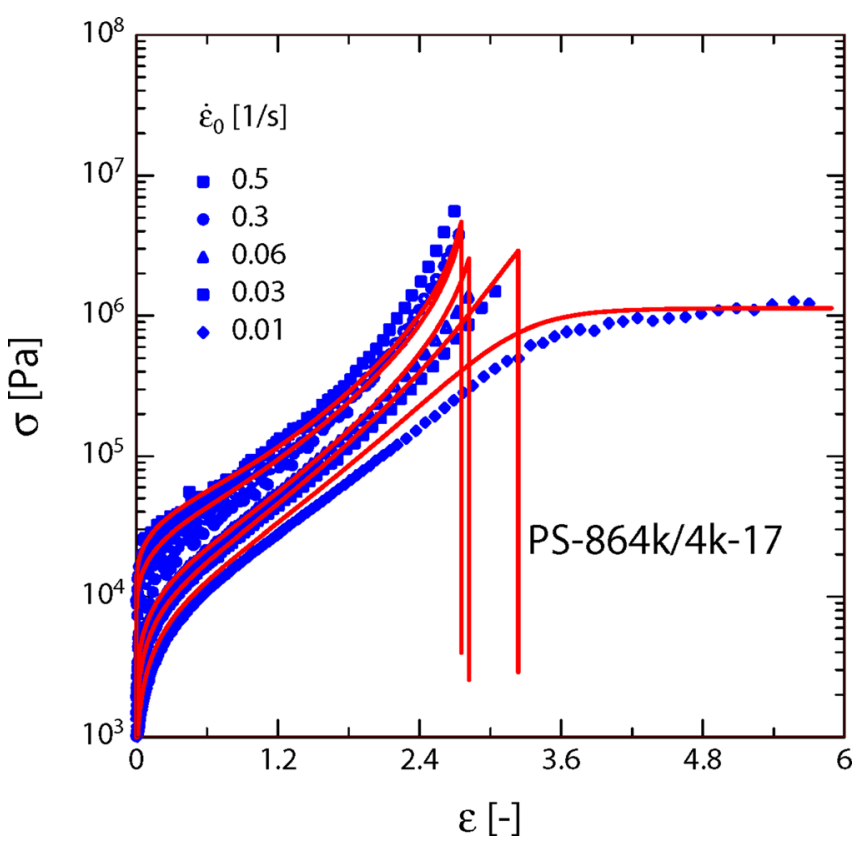

FIG. 11. Experimental data of stress as a function of Hencky strain for PS864k/4k-17 (symbols). Lines: Predictions of rupture by Eqs. (21), (25), and (26) for critical stretch $f_{c}=6$. Finite extensibility parameter $f_{m}=6.5$.

at rupture in Figs. 3 and 9, is documented in more detail in Fig. 11: While for strain rates of $0.03 \mathrm{~s}^{-1}$ and larger, fracture is observed and predicted, the polymer liquid can be extended up to a Hencky strain of six without failure at a strain rate of $0.01 \mathrm{~s}^{-1}$.

Finite extensibility effects are also clearly visible for the solution PS-545k/1k-52 (Fig. 12). Compared to Fig. 6, much better agreement between data and predictions can be obtained for a critical stretch at break of $f_{c}=5$ in combination with a finite extensibility parameter of $f_{m}=6.5$, which is

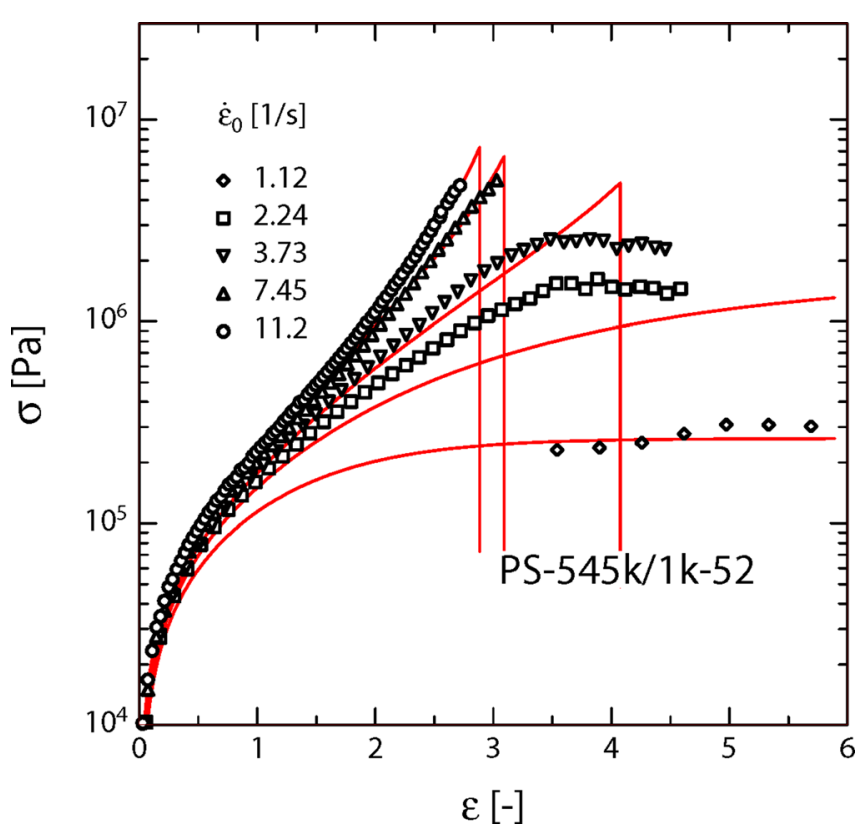

FIG. 12. Stress-strain data of PS-545k/1k-52 (symbols). Predictions by Eqs. (21), (25), and (26) with $f_{c}=5$ (lines). Finite extensibility parameter $f_{m}=6.5$. 


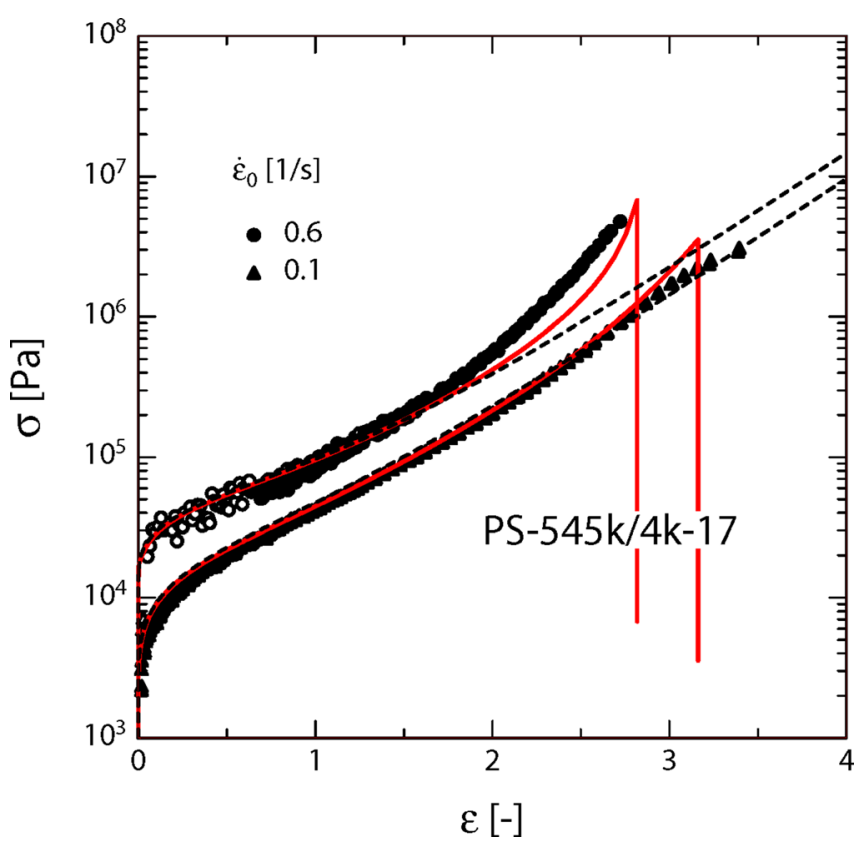

FIG. 13. Stress-strain data of PS-545k/4k-17 (symbols). Predictions of rupture by Eqs. (21), (25), and (26) with $f_{c}=6$ (lines). Finite extensibility parameter $f_{m}=6.5$.

close to the maximal stretch expected from the relation $\lambda_{m} \cong 5 \varphi^{-1 / 2}=6.9$. Interestingly, the same polymer PS$545 \mathrm{k}$ dissolved in $4 \mathrm{k}$ OS at a volume fraction of $17 \%$ (PS$545 \mathrm{k} / 4 \mathrm{k}-17$ ) also shows a considerable non-Gaussian effect in the stress at a strain rate of $0.6 \mathrm{~s}^{-1}$, albeit fracture occurs at a critical stretch of $f_{c}=6$ (Fig. 13). Table II summarizes the results for maximal stretch according to the relation $\lambda_{m} \cong 5 \varphi^{-1 / 2}$ and the critical stretch $f_{c}$ found when considering non-Gaussian effects.

Finite extensibility is associated with a stretch energy function, which increases more strongly with stretch $f$ than predicted by Eq. (7), and diverges at the finite extensibility parameter $f_{m}$. Adapting the empirical strain energy function proposed by Gent [71] to a corresponding stretch energy function for finite extensibility, $w_{f}$, results in

$$
w_{f}=-w_{e q} f_{m}^{2} \ln \left(1-\frac{f^{2}}{f_{m}^{2}}\right),
$$

which reduces to Eq. (7) in the limit of $f_{m} \rightarrow \infty$ and diverges at $f=f_{m}$. The critical stretch at rupture $f_{c}$ is reached when $w_{f}=U$, i.e., $f_{c}$ is given by

TABLE II. Comparison of maximum stretch $\lambda_{m}$ and critical stretch $f_{c}$.

\begin{tabular}{lccccc}
\hline \hline Sample name & $\begin{array}{c}M_{w} \text { of PS } \\
(\mathrm{kg} / \mathrm{mol})\end{array}$ & $\begin{array}{c}\text { Oligomeric } \\
\text { styrene } \\
(\mathrm{kg} / \mathrm{mol})\end{array}$ & $\begin{array}{c}\text { PS } \\
(\text { wt. \%) }\end{array}$ & $\begin{array}{c}\text { Maximum } \\
\text { stretch } \lambda_{m}\end{array}$ & $\begin{array}{c}\text { Critical } \\
\text { stretch } f_{c}\end{array}$ \\
\hline PS-864k/4k-17 & 864 & 4 & 17 & 12.1 & 6 \\
PS-864k/4k-33 & 864 & 4 & 33 & 8.7 & 5 \\
PS-545 k/1 k-52 & 545 & 1 & 52 & 6.9 & 5 \\
PS-545k/4k-17 & 545 & 4 & 17 & 12.1 & 6 \\
PS-3280k/4k-13 & 3280 & 4 & 13 & 13.9 & 6 \\
\hline \hline
\end{tabular}

$$
f_{c}=f_{m} \sqrt{1-\operatorname{ecp}\left(\frac{-U / 3 k T}{f_{m}^{2}}\right)} .
$$

For $f_{m}=6.5$, a value of $f_{c}=4.9$ is found. This is in qualitative agreement with experimental evidence for the more concentrated solutions PS-545k/1k-52 and PS-864k/4k-33 with finite extensibilities $\lambda_{m}<9$ (see Table II), and indicates a partial transition from the entropic to the enthalpic fracture mode due to finite extensibility effects, as the energy barrier of the $\mathrm{C}-\mathrm{C}$ bonds is reduced due to stretch of the bonds.

\section{COMPARISON TO DATA OF A POLYDISPERSE PS MELT}

Figure 14 presents data of a polydisperse PS melt as reported by Huang et al. [21] and Huang [42]. Due to its polydispersity (see Table I), a maximum stretch $\lambda_{m}>5$ can be expected because of dilution by low molar mass components. Brittle fracture is observed experimentally for strain rates of 0.6 [21], $0.1,0.06$, and $0.03 \mathrm{~s}^{-1}$ [42]. Although the MSF model used here is best suited for monodisperse polymer melts and solutions with a dominant relaxation mode, agreement of model predictions of Eqs. (20), (25), and (26) using a rupture stretch of $f_{c}=5$ and a finite extensibility parameter of $f_{m}=6.5$ is nearly quantitative. At strain rates of $0.01 \mathrm{~s}^{-1}$ and lower, steady-state elongation is predicted at large Hencky strains in agreement with experimental evidence. Deviations between model and data at lower strain rates are caused by the polydispersity of the melt.

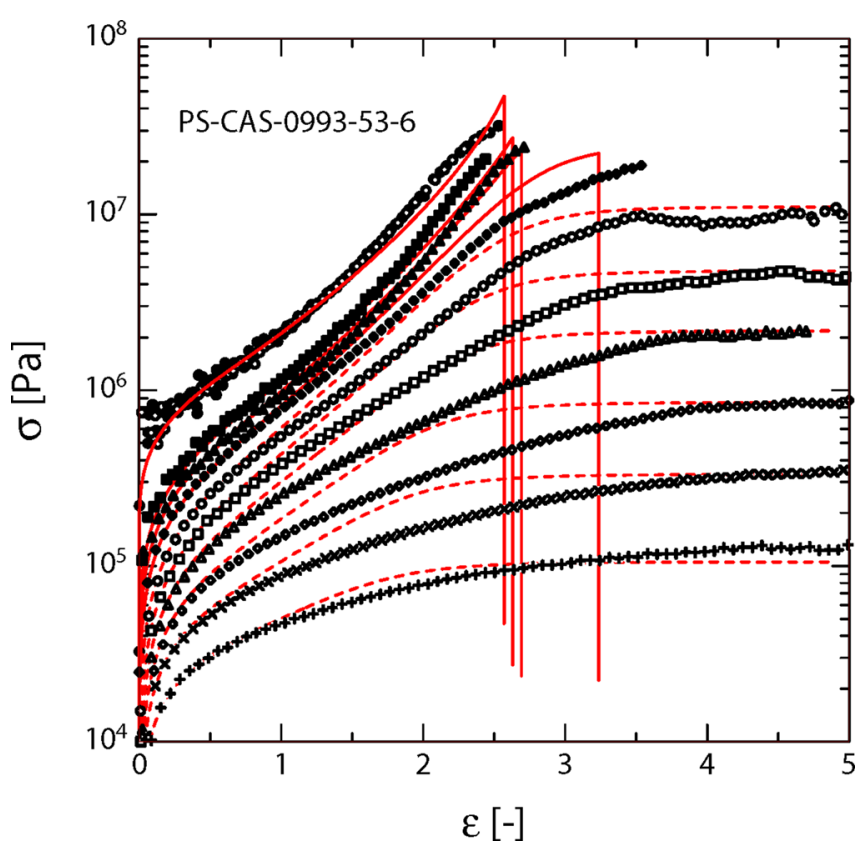

FIG. 14. Stress-strain data of PS melt (symbols). Predictions of extensional stress and rupture of PS melt as a function of Hencky strain by Eqs. (20), (25), and (26) with $f\left(t, t^{\prime}\right)$ limited to $f_{c}=5$ and $f_{m}=6.5$ (solid lines). Strain rates from top to bottom: $0.6,0.1,0.06,0.03,0.01,0.003,0.001,0.0003$, 0.0001 , and $0.00003 \mathrm{~s}^{-1}$. 


\section{CONCLUSIONS}

A physical explanation for the deterministic brittle fracture of entangled polymer solutions and melts has been presented. Crack initiation is caused by rupture of primary $\mathrm{C}-\mathrm{C}$ bonds. Assuming Gaussian chain statistics, a $\mathrm{C}-\mathrm{C}$ bond ruptures when due to molecular stretch $f$, the total strain energy $w_{s}=$ $3 k T f^{2}$ of an entanglement segment reaches the bond energy $U$ of a covalent $\mathrm{C}-\mathrm{C}$ bond. Thermal fluctuations will lead to a short-time concentration of the strain energy on one $\mathrm{C}-\mathrm{C}$ bond of the entangled chain segment, and the bond ruptures. This limits the maximum stretch of entanglement segments to a critical stretch of $f_{c} \leq \sqrt{U / 3 k T} \cong 6$. Available experimental data of solutions of nearly monodisperse PSs dissolved in OS and of a well characterized polydisperse PS melt are in general agreement with this entropic fracture model.

In contrast, the enthalpic fracture hypothesis of Lake and Thomas [36] as modified by Mazich and Samus [39], who assumed that a chain will only rupture, when it is fully stretched and the strain energy $w_{c}=N E_{\mathrm{C}-\mathrm{C}}$ corresponding to the bond energy of all $N \mathrm{C}-\mathrm{C}$ bonds in an entanglement segment is dissipated, predicts much higher strains and stresses at rupture than observed for entangled polymer liquids.

However, finite extensibility effects are visible in some of the data by a stress increasing over-proportionally with the square of the average stretch. This means that due to the stretch of $\mathrm{C}-\mathrm{C}$ bonds, the strain energy of the chain contains a certain fraction of enthalpic energy, which reduces the energy barrier of the $\mathrm{C}-\mathrm{C}$ bonds and leads to a critical fracture stretch of $f_{c}<6$. For the more concentrated solutions PS-545k/1k-52 and PS-864k/4k-33 and a PS melt with finite extensibilities $\lambda_{m}<9$, a critical stretch of $f_{c} \cong 5$ is found, indicating a partial transition from the entropic to the enthalpic fracture mode, due to a reduction of the energy barrier of the $\mathrm{C}-\mathrm{C}$ bond by stretching of the bond.

We may expect that a cross-over exists from the fracture criterion of well-cured rubbers to the fracture of polymer melts and solutions: For rubbers with $N_{x K} \leq N_{e M}$, where $N_{x K}$ is the number of Kuhn steps between two crosslinks and $N_{e M}$ the number of Kuhn steps in an entanglement segment of the corresponding melt, the maximal stretch is $\lambda_{m} \cong N_{x K}^{1 / 2}$, which is usually less than 6 and fracture does not occur by thermal fluctuations, but when the tension in the chain exceeds the rupture force of $\mathrm{C}-\mathrm{C}$ bonds (enthalpic fracture). On the other hand for polymer gels, melts, and solutions with maximal stretch $\lambda_{m} \cong\left(N_{e M} \varphi^{-1}\right)^{1 / 2} \geq 6$, the stretch at rupture is limited by thermal fluctuations (entropic fracture) to $f_{c}=\sqrt{U / 3 k T} \cong 6$, which represents an upper limit for the critical stretch at rupture for both entangled polymer liquids and elastomers. Entropic fracture of polymer liquids with maximal stretch $\lambda_{m}<9$ is influenced by finite extensibility effects, as the $\mathrm{C}-\mathrm{C}$ bonds are stretched and therefore the energy required for chain scission is reduced, and these polymer liquids fracture at a critical molecular stretch $f_{c}<6$.

\section{ACKNOWLEDGMENT}

Q.H. would like to acknowledge financial support from the Aage og Johanne Louis-Hansen Foundation.

\section{References}

[1] Andrade, R., and J. Maia, "A study on the flow, failure, and rupture mechanisms of low-density polyethylene in controlled-stress uniaxial extensional flow," J. Rheol. 55, 925-937 (2011).

[2] Joshi, Y. M., and M. M. Denn, "Failure and recovery of entangled polymer melts in elongational flow," Rheol. Rev. 2004, 1-17.

[3] Reiner, M., and A. Freudenthal, "Failure of a material showing creep (a dynamical theory of strength)," in Proceedings of the 5th International Congress on Applied Mechanics 1938, pp. 228-233 [reprinted in M. Reiner, Selected Papers (Elsevier, Amsterdam, 1975), pp. 141-148].

[4] Considère, M., "Memoire sur l'emploi du fer et de l'acier dans les constructions," in Annales des Ponts et Chausées (Ch. Dunod, Paris, 1885), Vol. 9, pp. 574-605.

[5] McKinley, G. H., and O. Hassager, "The Considere condition and rapid stretching of linear and branched polymer melts," J. Rheol. 43, 1195-1212 (1999).

[6] Doi, M., and S. F. Edwards, "Dynamics of concentrated polymer systems. Part 4: Rheological properties," J. Chem. Soc. 75, 38-54 (1979).

[7] McLeish, T. C. B., and R. G. Larson, "Molecular constitutive equations for a class of branched polymers: The pom-pom polymer," J. Rheol. 42, 81-110 (1998).

[8] Vinogradov, G., "Viscoelasticity and fracture phenomenon in uniaxial extension of high-molecular linear polymers," Rheol. Acta 14, 942-954 (1975).

[9] Vinogradov, G., "Ultimate regimes of deformation of linear flexible chain fluid polymers," Polymer 18, 1275-1285 (1977).

[10] Vinogradov, G., V. Dreval, E. Borisenkova, M. Kurbanaliev, and V. Shalganova, "Uniaxial extension of linear flexible-chain polymers in an extremely broad range of stresses and strain rates," Rheol. Acta $\mathbf{2 0}$, 433-442 (1981).

[11] Vinogradov, G., A. Y. Malkin, and V. Volosevitch, "Some fundamental problems in viscoelastic behavior of polymers in shear and extension,” J. Appl. Polym. Sci.: Appl. Polym. Symp. 27, 47-59 (1975).

[12] Vinogradov, G., V. Volosevitch, V. Shatalov, and V. Yudin, "Flow, high-elastic (recoverable) deformation, and rupture of uncured high molecular weight linear polymers in uniaxial extension," J. Polym. Sci.: Polym. Phys. Ed. 13, 1721-1735 (1975).

[13] Vinogradov, G., and A. Y. Malkin, Rheology of polymers: Viscoelasticity and flow of polymers (Springer, 1980).

[14] Vinogradov, G., Y. G. Yanovskii, E. Borisenkova, B. Yarlykov, and G. Berezhnaya, "Viscoelastic properties and flow of narrow distribution polybutadienes and polyisoprenes," J. Polym. Sci. Part A-2: Polym. Phys. 10, 1061-1084 (1972).

[15] Malkin, A. Y., and C. Petrie, "Some conditions for rupture of polymer liquids in extension," J. Rheol. 41, 1-25 (1997).

[16] Wang, Y., and S.-Q. Wang, "Rupture in rapid uniaxial extension of linear entangled melts," Rheol. Acta 49, 1179-1185 (2010).

[17] Wang, Y., S. Cheng, and S.-Q. Wang, "Basic characteristics of uniaxial extension rheology: Comparing monodisperse and bidisperse polymer melts," J. Rheol. 55, 1247-1270 (2011).

[18] Wang, Y., and S.-Q. Wang, "Salient features in uniaxial extension of polymer melts and solutions: Progressive loss of entanglements, yielding, non-Gaussian stretching, and rupture," Macromolecules 44, 5427-5435 (2011).

[19] Wang, Y., and S.-Q. Wang, "From elastic deformation to terminal flow of a monodisperse entangled melt in uniaxial extension," J. Rheol. 52, 1275-1290 (2008).

[20] Wang, Y., and S.-Q. Wang, "Exploring stress overshoot phenomenon upon startup deformation of entangled linear polymeric liquids," J. Rheol. 53, 1389-1401 (2009). 
[21] Huang, Q., N. J. Alvarez, A. Shabbir, and O. Hassager, "Multiple cracks propagate simultaneously in polymer liquids in tension," Phys. Rev. Lett. 117, 087801 (2016).

[22] Huang, Q., and O. Hassager, "Polymer liquids fracture like solids," Soft Matter 13, 3470-3474 (2017).

[23] Huang, Q., M. Mangnus, N. J. Alvarez, R. Koopmans, and O. Hassager, "A new look at extensional rheology of low-density polyethylene," Rheol. Acta 55, 343-350 (2016).

[24] Joshi, Y. M., and M. M. Denn, "Rupture of entangled polymeric liquids in elongational flow," J. Rheol. 47, 291-298 (2003).

[25] Joshi, Y. M., and M. M. Denn, "Rupture of entangled polymeric liquids in elongational flow with dissipation," J. Rheol. 48, 591-598 (2004).

[26] Wang, S.-Q., S. Ravindranath, Y. Wang, and P. Boukany, "New theoretical considerations in polymer rheology: Elastic breakdown of chain entanglement network,” J. Chem. Phys. 127, 064903 (2007).

[27] Zhu, X., and S.-Q. Wang, "Mechanisms for different failure modes in startup uniaxial extension: Tensile (rupture-like) failure and necking," J. Rheol. 57, 223-248 (2013).

[28] Brochard, F., and P. De Gennes, "Shear-dependent slippage at a polymer/solid interface,” Langmuir 8, 3033-3037 (1992).

[29] Griffith, A. A., "The phenomena of rupture and flow in solids," Philos. Trans. R. Soc. London Ser. A 221, 163-198 (1921).

[30] Pomeau, Y., "Brisure spontanée de cristaux bidimensionels courbés," C.R. Acad. Sci., Ser. 2 Mec. Phys. 314(6), 553-556 (1992).

[31] Tabuteau, H., S. Mora, M. Ciccotti, C.-Y. Hui, and C. Ligoure, "Propagation of a brittle fracture in a viscoelastic fluid," Soft Matter 7, 9474-9483 (2011).

[32] Tabuteau, H., S. Mora, G. Porte, M. Abkarian, and C. Ligoure, "Microscopic mechanisms of the brittleness of viscoelastic fluids," Phys. Rev. Lett. 102, 155501 (2009).

[33] Ligoure, C., and S. Mora, "Fractures in complex fluids: The case of transient networks," Rheol. Acta 52, 91-114 (2013).

[34] Lake, G., and P. Lindley, "Cut growth and fatigue of rubbers. II. Experiments on a noncrystallizing rubber," J. Appl. Polym. Sci. 8, 707-721 (1964).

[35] Lake, G., and P. Lindley, "The mechanical fatigue limit for rubber," J. Appl. Polym. Sci. 9, 1233-1251 (1965).

[36] Lake, G., and A. Thomas, "The strength of highly elastic materials," Proc. R. Soc. Lond. A 300, 108-119 (1967).

[37] Bhowmick, A., A. Gent, and C. Pulford, "Tear strength of elastomers under threshold conditions," Rubber Chem. Technol. 56, 226-232 (1983).

[38] Akagi, Y., H. Sakurai, J. P. Gong, U.-I. Chung, and T. Sakai, "Fracture energy of polymer gels with controlled network structures," J. Chem. Phys. 139, 144905 (2013).

[39] Mazich, K. A., and M. Samus, "Role of entanglement couplings in threshold fracture of a rubber network," Macromolecules 23, 2478-2483 (1990).

[40] Grandbois, M., M. Beyer, M. Rief, H. Clausen-Schaumann, and H. E. Gaub, "How strong is a covalent bond?," Science 283, 1727-1730 (1999).

[41] Hanson, D. E., and R. L. Martin, "How far can a rubber molecule stretch before breaking? Ab initio study of tensile elasticity and failure in single-molecule polyisoprene and polybutadiene," J. Chem. Phys. 130, 064903 (2009).

[42] Huang, Q., "Molecular rheology of complex fluids," Ph.D. thesis, Technical University of Denmark, Copenhagen, 2013.

[43] Huang, Q., N. J. Alvarez, Y. Matsumiya, H. K. Rasmussen, H. Watanabe, and O. Hassager, "Extensional rheology of entangled polystyrene solutions suggests importance of nematic interactions," ACS Macro Lett. 2, 741-744 (2013).
[44] Q. Huang, and H. K. Rasmussen, "Stress relaxation following uniaxial extension of polystyrene melt and oligomer dilutions," J. Rheol. 60, 465-471 (2016).

[45] Huang, Q., L. Hengeller, N. J. Alvarez, and O. Hassager, "Bridging the gap between polymer melts and solutions in extensional rheology," Macromolecules 48, 4158-4163 (2015).

[46] Marrucci, G., and J. J. Hermans, "Nonlinear viscoelasticity of concentrated polymeric liquids," Macromolecules 13, 380-387 (1980).

[47] De Gennes, P., "Coil-stretch transition of dilute flexible polymers under ultrahigh velocity gradients," J. Chem. Phys. 60, 5030-5042 (1974).

[48] Marrucci, G., "Molecular modelling of flows of concentrated polymers," in Transport Phenomena in Polymeric Systems (Wiley, New York, 1989).

[49] Wagner, M. H., P. Rubio, and H. Bastian, "The molecular stress function model for polydisperse polymer melts with dissipative convective constraint release," J. Rheol. 45, 1387-1412 (2001).

[50] Wagner, M. H., and J. Schaeffer, "Nonlinear strain measures for general biaxial extension of polymer melts," J. Rheol. 36, 1-26 (1992).

[51] Wagner, M. H., and J. Schaeffer, "Rubbers and polymer melts: Universal aspects of nonlinear stress-strain relations," J. Rheol. 37, 643-661 (1993).

[52] Wagner, M. H., and J. Schaeffer, "Assessment of nonlinear strain measures for extensional and shearing flows of polymer melts," Rheol. Acta 33, 506-516 (1994).

[53] Doi, M., and S. F. Edwards, The Theory of Polymer Dynamics (Oxford University, Oxford, 1986).

[54] Pearson, D. S., A. D. Kiss, L. J. Fetters, and M. Doi, "Flow-induced birefringence of concentrated polyisoprene solutions," J. Rheol. 33, 517-535 (1989).

[55] Bach, A., K. Almdal, H. K. Rasmussen, and O. Hassager, "Elongational viscosity of narrow molar mass distribution polystyrene," Macromolecules 36, 5174-5179 (2003).

[56] Wagner, M. H., S. Kheirandish, and O. Hassager, "Quantitative prediction of transient and steady-state elongational viscosity of nearly monodisperse polystyrene melts," J. Rheol. 49, 1317-1327 (2005).

[57] Marrucci, G., and G. Ianniruberto, "Interchain pressure effect in extensional flows of entangled polymer melts," Macromolecules 37, 3934-3942 (2004).

[58] Wagner, M. H., "The effect of dynamic tube dilation on chain stretch in nonlinear polymer melt rheology," J. Non-Newtonian Fluid Mech. 166, 915-924 (2011).

[59] Wagner, M. H., "Scaling relations for elongational flow of polystyrene melts and concentrated solutions of polystyrene in oligomeric styrene," Rheol. Acta 53, 765-777 (2014).

[60] Wagner, M. H., "An extended interchain tube pressure model for elongational flow of polystyrene melts and concentrated solutions," J. NonNewtonian Fluid Mech. 222, 121-131 (2015).

[61] Huang, Q., O. Mednova, H. K. Rasmussen, N. J. Alvarez, A. L. Skov, K. Almdal, and O. Hassager, "Concentrated polymer solutions are different from melts: Role of entanglement molecular weight," Macromolecules 46, 5026-5035 (2013).

[62] Fang, J., M. Kröger, and H. C. Öttinger, "A thermodynamically admissible reptation model for fast flows of entangled polymers. II. Model predictions for shear and extensional flows," J. Rheol. 44, 1293-1317 (2000).

[63] Graessley, W. W., Polymeric Liquids and Networks: Structure and Properties (Garland Science, New York, 2003).

[64] Fetters, L., D. Lohse, D. Richter, T. Witten, and A. Zirkel, "Connection between polymer molecular weight, density, chain dimensions, and melt viscoelastic properties," Macromolecules 27, 4639-4647 (1994). 
[65] Fetters, L. J., D. J. Lohse, and W. W. Graessley, "Chain dimensions and entanglement spacings in dense macromolecular systems," J. Polym. Sci. Part B: Polym. Phys. 37, 1023-1033 (1999).

[66] van Meerveld, J., "Validity of the linear stress optical rule in mono-, bi-and polydisperse systems of entangled linear chains," J. NonNewtonian Fluid Mech. 123, 259-267 (2004).

[67] Fetters, L. J., D. J. Lohse, S. T. Milner, and W. W. Graessley, "Packing length influence in linear polymer melts on the entanglement, critical, and reptation molecular weights," Macromolecules 32, 6847-6851 (1999).
[68] Rolón-Garrido, V. H., M. H. Wagner, C. Luap, and T. Schweizer, "Modeling non-Gaussian extensibility effects in elongation of nearly monodisperse polystyrene melts," J. Rheol. 50, 327-340 (2006).

[69] Bird, R. B., R. C. Armstrong, and O. Hassager, Dynamics of Polymeric Liquids (Wiley, New York, 1987).

[70] Ye, X., and T. Sridhar, "Effects of the polydispersity on rheological properties of entangled polystyrene solutions," Macromolecules 38, 3442-3449 (2005).

[71] Gent, A., "A new constitutive relation for rubber," Rubber Chem. Technol. 69, 59-61 (1996). 